\title{
ESTUDIO PRELIMINAR DE LA UTILIDAD DE UN PROGRAMA DE REALIDAD VIRTUAL CONTRA EL ESTIGMA EN SALUD MENTAL
}

\author{
Adolfo J. Cangas \\ Universidad de Almería \\ ajcangas@ual.es \\ Blanca Galván \\ Universidad de Almería
}

Recepción Artículo: 26 febrero 2020

Admisión Evaluación: 4 marzo 2020

Informe Evaluador 1: 13 marzo 2020

Informe Evaluador 2: 15 Marzo 2020

Aprobación Publicación: 20 abril 2020

\section{RESUMEN}

En el presente estudio se presentan los resultados de la aplicación de un nuevo programa de realidad virtual, diseñado para sensibilizar sobre el estigma hacia las personas con problemas de salud mental. Específicamente, el software, denominado Inclúyete $V R$, presenta primeramente una persona que está sufriendo diversas alucinaciones (representado gráficamente por "monstruos") y, posteriormente, diferentes programas de rehabilitación psicosocial que se utilizan para la inclusión social de personas con trastorno mental grave. Inclúyete VR se aplicó a una muestra 12 psicólogos que estaban cursando en la actualidad el Máster de Psicología General Sanitaria. Los resultados obtenidos mostraron que se redujo el estigma de un modo estadísticamente significativo en estos profesionales. Asimismo, valoraron con más de 8 puntos (sobre una escala de 1-10) el interés y utilidad del programa. Se discute la importancia de estos resultados y las líneas de investigación futuras.

Palabras clave: estigma; realidad virtual; trastornos mentales; salud mental; esquizofrenia

\section{ABSTRACT}

Preliminary study of the usefulness of a virtual reality programme against stigma in mental

health. This study shows the results of the application of a new virtual reality program, designed to raise awareness of stigma towards people with mental health problems. Specifically, the software, called Inclúyete VR, shows first a person who is suffering from hallucinations (graphically represented by "monsters") and, later, different psychosocial rehabilitation programs that are used for the social inclusion of people with severe mental disorder. A sample of 12 psychologists who were currently taking the Master of General Health Psychology use Inclúyete VR. The results obtained showed that stigma was reduced in a statistically significant way in these professionals. Likewise, they valued with more than 8 points (on a scale of 1-10) the interest and usefulness of the program. The importance of these results and future lines of research are discussed.

Keywords: stigma; virtual reality; mental disorders, mental health; schizophrenia 


\section{ESTUDIO PRELIMINAR DE LA UTILIDAD DE UN PROGRAMA DE REALIDAD VIRTUAL CONTRA EL ESTIGMA EN SALUD MENTAL}

\section{OBJETIVOS DE LA INVESTIGACIÓN}

El estigma social hacia las personas que padecen algún problema de salud mental es hoy en día una de las principales barreras en la recuperación de estas personas. Así, aunque se ha mejorado mucho en diferentes intervenciones clínicas, aún prevalece la idea, a pesar de múltiples estudios en contra, de que la mayoría de estas personas son violentas, incapaces de llevar una vida independiente, sin posibilidad de recuperación, etc, lo que genera en este colectivo un estrés adicional añadido, depresión y un aislamiento social importante (Corrigan y Watson, 2005; Fresán, Robles, de Benito, Saracco y Escamilla., 2010).

Estas ideas estigmatizantes no están presentes solo en la población general, sino que también en muchos casos se dan en los propios profesionales de la salud mental, de ahí la importancia de llevar a cabo también diferentes programas para sensibilizar sobre el estigma a estos profesionales (Granados-Gámez, López Rodríguez, Corral Granados, Márquez-Hernández, 2010; Hansson, Jormfeldt, Svedberg, Svensson, 2013; Horsfall, Cleary y Hunt, 2010; Mårtensson, Jacobsson, Engström, 2010; Wahly, Aroesty-Cohen, 2010).

Para la reducción del estigma se suele recurrir a diferentes estrategias que incluyen charlas, videos, carteles publicitarios, etc (Corrigan, River, et al., 2001; Longdon y Read, 2017). Lo que no ha sido tan común hasta el momento es recurrir a programas informáticos, quizá porque son más novedosos y su desarrollo requiere generalmente de la colaboración de programadores.

Particularmente, en 2017 se presentó el primer serious game (videojuego educativo) para trabajar el estigma con estudiantes (Cangas, Navarro, Aguilar-Parra, Ojeda, Cangas, Piedra y Gallego, 2017). El mismo, denominado Stigma-Stop, recrea a cuatro personas que sufren diferentes problemas de salud mental, como son esquizofrenia, trastorno bipolar, agorafobia y depresión, mostrando diferentes estrategias para ayudarles, a la vez que se pregunta a la persona si alguna vez se han sentido como los personajes y si creen que pueden ayudarles. Los resultados obtenidos mostraron una reducción importante del estigma tanto en estudiantes de enseñanzas medias como de universidad (Cangas, Navarro, Aguilar, Trigueros, Gallego, Zárate y Gregg, 2019; Mullor, Sayans, Cangas y Navarro, 2019).

Un paso más allá creemos que es utilizar programas de realidad virtual. A través de esta tecnología ya no solo se está viendo "en tercera persona" cómo se comporta un personaje (como ocurre en la simulación 3D o en los serious games), sino que es el propio participante "en primera persona" el protagonista de la acción, moviéndose e interaccionando por el espacio virtual. Este aspecto puede ser muy importante a la hora de tener mayor implicación personal en la experiencia (Hortensius, Neyret, Slater y Gelder, 2018).

Por otro lado, desde 2016, cuando aparecieron en el mercado las primeras gafas de realidad virtual de Oculus Rift, la reducción de su precio y la facilidad de uso hace que esta tecnología sea cada vez sea común, lo que favorece que probablemente en un futuro inmediato se generalice su uso de un modo notable.

En el presente trabajo mostramos los resultados de aplicar un programa de realidad virtual diseñado para reducir el estigma hacia las personas con trastorno mental grave. El mismo, que denominamos "Inclúyete VR" está inspirado en el proyecto socioeducativo "Inclúyete", donde usuarios de salud mental y estudiantes comparten clases en un programa de actividades deportivas y culturales, que sirve de inclusión social para las personas con problemas graves de salud mental y de reducción del estigma en los estudiantes, profesionales y voluntarios que participan en la experiencia (más información del programa en www.incluyete.blog).

En Inclúyete VR se muestra así, en una primera fase, cómo se puede sentir una persona que está pasando por dificultades personales importantes y está sufriendo alucinaciones y, en una segunda fase, la importancia de actividades socioeducativas en la recuperación. Todo ello con el doble objetivo, por un lado, de mostrar la "angustia" o sufrimiento de una persona que está pasando por un problema grave de salud mental y también diferentes vías que le ayudan en su recuperación.

En el presente estudio, hemos aplicado Inclúyete VRa una muestra de psicólogos, con un doble objetivo, analizar por un lado si contribuye a reducir el estigma en estas personas y, por otro, para analizar la valoración que hacen estos profesionales de su utilidad e interés. 


\section{PARTICIPANTES}

Participaron en el estudio un total de 12 psicólogos que estaban cursando el Máster de Psicología General Sanitaria en la Universidad de Almería y que de modo voluntario quisieron participar en la investigación. Se trata así de un muestreo por conveniencia. Los participantes no recibieron ninguna remuneración económica ni créditos extra por participar en el estudio.

En cuanto a las características sociodemográficas de la muestra, 10 eran mujeres y 2 hombres. La edad media fue de 26 años (D.T.=4,9). La mitad de la muestra (6 personas) indicaron que conocían 0 tenían relación (familiar, pareja 0 amigo/a íntimo) con alguna persona con trastorno mental grave. 4 señalaron que habían tenido algún encuentro esporádico con alguna persona, 2 indicaron que tenían algún conocido 0 amigo/a que tenía un trastorno mental grave y uno de ellos indicó que él mismo padecía un trastorno mental con estas características.

\section{INSTRUMENTOS}

- Inclúyete VR. Programa de realidad virtual desarrollado con UNITY donde el jugador se tiene que poner en el lugar de una persona que sufre un problema de salud mental. Para ello inicialmente se muestra un escenario con "monstruos" (que representan alucinaciones) donde el jugador puede matarlos, pero llega un momento que el número de ellos y la velocidad a la que actúan hace imposible este propósito y aparece un "monstruo grande" del que solo queda huir. A continuación, un personaje ofrece al jugador alcohol y al beberlo el juego se funde a negro y aparece el sonido de ambulancias.

En la segunda parte del programa, la persona está en un "Centro de crisis" donde hay otras personas que comentan que han pasado por situaciones similares y puede ir por diferentes salas y hacer distintas actividades. En concreto, hay un perro al que puede acariciar y mueve la cola. Existe un gimnasio con una canasta donde el participante puede jugar y encestar. Hay una sala con un lienzo donde puede pintar. Un taller con piezas de un ordenador, donde tiene que ensamblarlas. Un estudio de radio donde existe un mezclador de sonidos y puede subirlo y bajarlo y, por último, un jardín con maceteras de tomates que puede regar y crecen los frutos.

Esta sala está diseñada en formato de escape room, donde después de hacer cada actividad propuesta la persona va ganando una estrella y al final puede salir. Igualmente, una vez que va realizando cada una de las actividades aparece un mensaje de la utilidad de las diferentes tareas, en concreto, se comenta: el apoyo entre iguales nos ayuda a sentirnos comprendidos, Ios animales también pueden mejorar nuestro bienestar personal, la actividad física es un aliado de la salud mental, a través del arte podemos compartir nuestras emociones, el trabajo nos ayuda a valorarnos y las rutinas nos ayudan a organizarnos. En la figura 1 se muestra algunas capturas de pantalla de este programa. Más información del programa se puede obtener en su página web (https://incluyete.blog/incluyete-vr/) 


\section{ESTUDIO PRELIMINAR DE LA UTILIDAD DE UN PROGRAMA DE REALIDAD VIRTUAL CONTRA EL ESTIGMA EN SALUD MENTAL}

Figura 1. Capturas de pantalla de Inclúyete VR

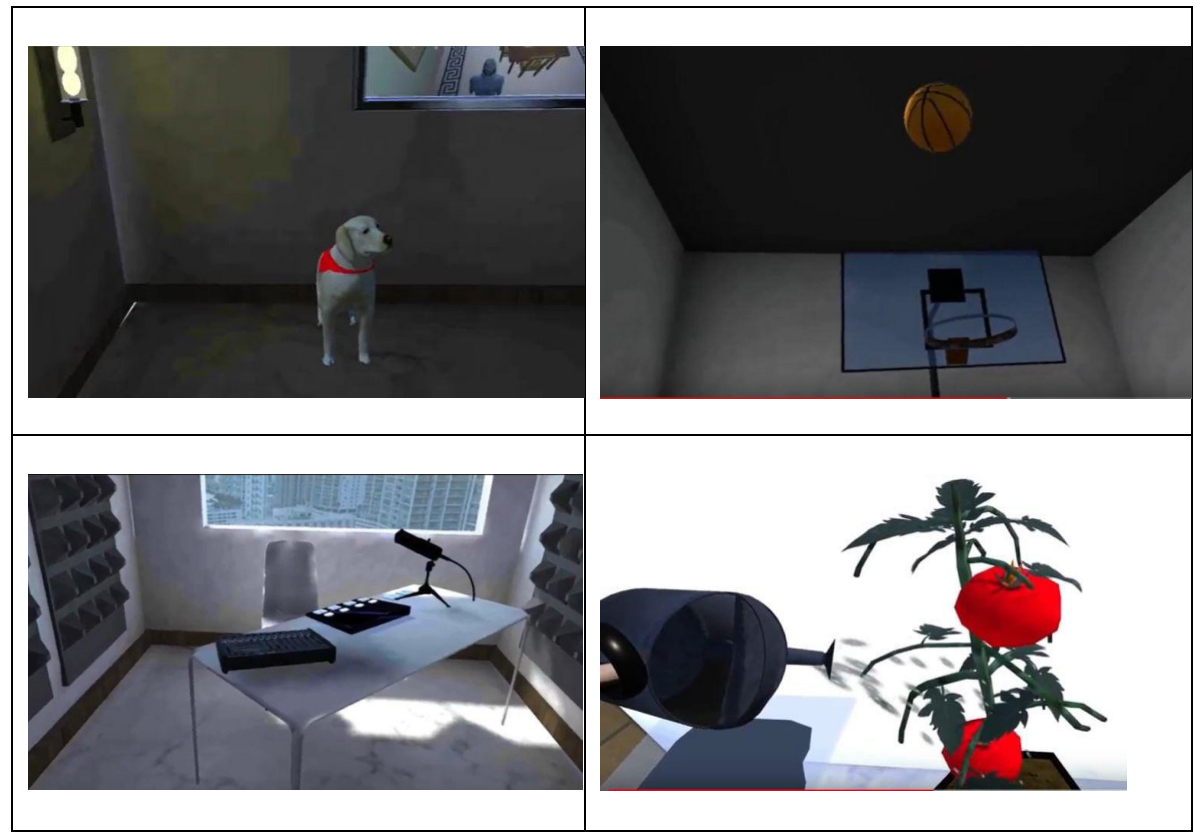

Cuestionario Atribucional (AQ27). Se trata de un cuestionario muy utilizado para medir estigma en la población general, donde se presenta una situación de una persona diagnosticada con esquizofrenia y se le pregunta diferencias creencias y actitudes sobre esta persona en una escala Likert de 9 puntos. Para el presente estudio utilizaremos la versión abreviada de 14 ítems validada recientemente al castellano por Saavedra, Arias, Corrigan y López (2020), la cual ha obtenido adecuadas propiedades psicométricas en sus cuatro factores (Miedo-peligrosidad=0, 88; Ausencia de solidaridad=0,837; Coerción=0,864 y Evitación=0,758).

Cuestionario de valoración de Inclúyete-VR. Se trata de un instrumento diseñado ad hoc para la presente investigación, donde los participantes tienen que puntuar de 1-10 el interés y la utilidad del programa de realidad en la reducción del estigma, así como la experiencia y familiaridad que tienen con los programas de realidad virtual. En la tabla1 se muestra las diferentes preguntas que contiene.

\section{PROCEDIMIENTO}

En el máster de Psicología General Sanitaria se ofreció la posibilidad de participar en una investigación para probar un software de realidad virtual. Los voluntarios fueron citados

individualmente para llevar a cabo la sesión. Inicialmente rellenaron el AAQ-27 y a continuación participaban en Inclúyete VR; a continuación, cumplimentaron de nuevo el AAQ-27 y realizaron el cuestionario diseñado ad hoc para para valorar la experiencia.

\section{RESULTADOS}

En primer lugar, en cuanto a las puntuaciones en estigma, cabe señalar que la muestra partía de puntuaciones medio-bajas en esta variable antes de pasar por la intervención. En concreto, la media en la puntuación total en el AAQ-27 fue de 25,33 puntos ( $D . T_{.}=9,86$ ) (en un rango que puede ir desde 14 hasta 126 puntos) y en cuanto al número de participantes, tres de ellos estaban por debajo de 20 puntos (figura 1). 
En la evaluación postratamiento se obtuvo una reducción total de 3,17 puntos, siendo estadísticamente significativa esta diferencia mediante la t de Student para muestras relacionadas ( $t=2,61 ; p<0,05)$. Asimismo, cabe señalar que siete participantes pasaron a estar por debajo de 20 puntos en la AAQ-27 después de la intervención (figura 1).

En cuanto a los factores de esta escala, la reducción mayor se produjo en peligrosidad- miedo (1,91 puntos de diferencia), a continuación, en Evitación (0,75 puntos), siendo en la Falta de solidaridad y Coerción el cambio menor, en concreto de 0,25 puntos (debido probablemente a que las puntuaciones iniciales de las que partían los participantes eran muy bajas en estas dos dimensiones, en concreto de 5,5 y 3,4 puntos respectivamente).

Haciendo un análisis individualizado de los datos, 8 participantes redujeron su puntuación en estigma (participantes 1,3,5,6,7,8, 11 y 12); uno sube (participante 2) y tres se mantienen en la misma puntuación (participantes 4,9 y 10) que corresponden precisamente a los que tenían puntuaciones más bajas en la evaluación de partida (por debajo de 20 puntos) (figura 2).

Figura 2. Puntuaciones en el AAQ-27 de cada participante antes (azul) y después de la intervención (marrón)

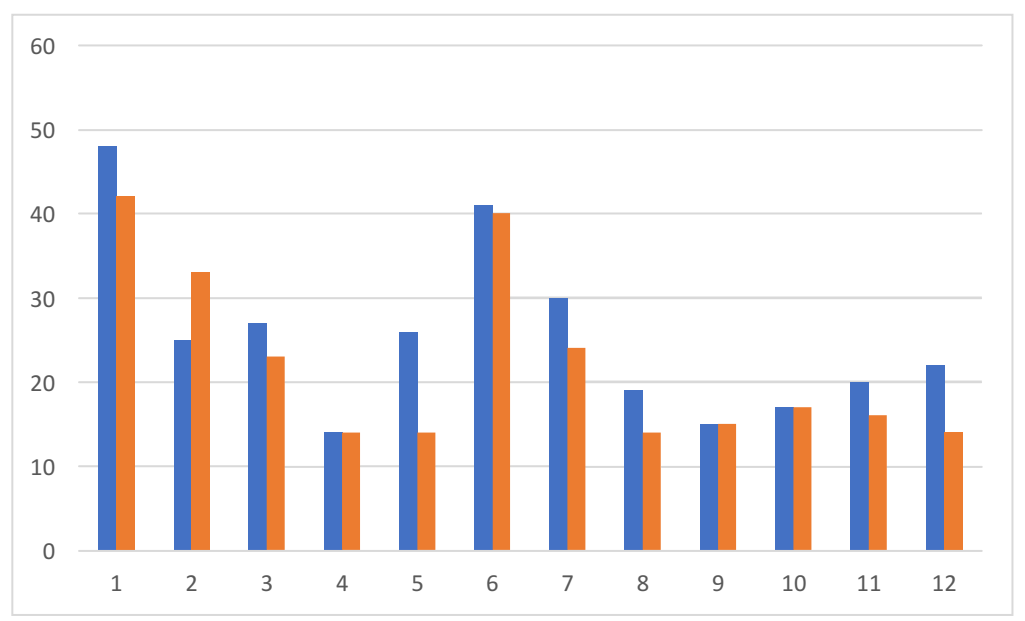

Por lo que se refiere a la valoración que hicieron del programa, en la Tabla 1 se muestra la puntuación en cada ítem (en un rango que va de 1-10). Cabe destacar que todas las puntuaciones estuvieron por encima de los ocho puntos. 
Tabla 1. Valoración de Inclúyete VR

\begin{tabular}{|l|c|}
\hline \multicolumn{1}{|c|}{ Item } & $\begin{array}{c}\text { Media } \\
\text { (D.T.) }\end{array}$ \\
\hline $\begin{array}{l}\text { ¿En qué grado crees que esta experiencia de realidad virtual puede } \\
\text { ayudar a que los estudiantes en general tengan más interés por conocer } \\
\text { aspectos relacionados con la salud mental? }\end{array}$ & $8,5(1,5)$ \\
\hline $\begin{array}{l}\text { ¿En qué grado consideras que esta experiencia de realidad virtual puede } \\
\text { ayudar a otras personas a mejorar su comprensión del proceso de } \\
\text { recuperación en personas con problemas graves de salud mental? }\end{array}$ & $8,5(1,3)$ \\
\hline $\begin{array}{l}\text { ¿En qué grado consideras que esta experiencia de realidad virtual ha sido } \\
\text { educativa, en general, sobre los problemas graves de salud mental? }\end{array}$ & $8,1(1,9)$ \\
\hline $\begin{array}{l}\text { ¿En qué grado consideras que este programa de realidad virtual puede } \\
\text { hacer a quienes participen más empáticos hacia las personas con } \\
\text { problemas graves de salud mental? }\end{array}$ & $8,3(1)$ \\
\hline $\begin{array}{l}\text { ¿En qué grado consideras que este programa de realidad virtual puede } \\
\text { ser útil para favorecer la inclusión social de las personas con problemas } \\
\text { graves de salud mental? }\end{array}$ & $8,3(1,1)$ \\
\hline $\begin{array}{l}\text { ¿Qué nivel de inmersión o sensación de estar en la realidad has sentido } \\
\text { durante la experiencia? }\end{array}$ & $8,4(0,8)$ \\
\hline ¿Cuánto de entretenido ha sido para ti esta simulación de realidad virtual & $8,9(1,9)$ \\
\hline $\begin{array}{l}\text { ¿Recomendarías a un amigo/ que experimente este programa de realidad } \\
\text { virtual? }\end{array}$ & $9,2(0,3)$ \\
\hline
\end{tabular}

\section{DISCUSIÓN}

Inclúyete VR es el primer programa de realidad virtual encaminado a sensibilizar sobre el estigma a los participantes. En el presente estudio, se ha llevado a cabo la aplicación con una muestra de psicólogos que estaban cursando el Máster de Psicología General Sanitaria. En primer lugar, cabe destacar que los profesionales bajaron su puntuación total en el estigma en el cuestionario AQ-27, particularmente en los factores de peligrosidadmiedo y de evitación.

De todos modos, cabe señalar que la puntuación inicial no era muy alta, quizá debida la formación que los profesionales tenían en el propio Máster que, aunque no tienen un módulo específico, sobre el estigma en salud mental, sí que tienen materias relacionadas con el trastorno mental grave, donde se toca este tema transversalmente. Diversos estudios muestran de hecho que el estigma entre los profesionales sanitarios es bastante común (Granados-Gámez, López Rodríguez, Corral Granados, Márquez-Hernández, 2010; Hansson, Jormfeldt, Svedberg, Svensson, 2013; Horsfall, Cleary y Hunt, 2010; Mårtensson, Jacobsson, Engström, 2010; Wahly, Aroesty-Cohen, 2010).

Por lo que se refiere a la valoración que hacen en sí del programa, cabe destacar que las puntuaciones en general fueron altas, indicando con valores superiores a ocho puntos que el programa puede ayudar a tener mayor interés por la salud mental, a comprender mejor los procesos de recuperación, a ser más empáticos con los usuarios de salud mental, y a favorecer la inclusión social. En cuanto la experiencia en sí, señalan también con valores altos que la experiencia la ven altamente inmersiva, entretenida y que recomendarían a un amigo probarla.

Entre las limitaciones del estudio cabe destacar en primer lugar la muestra baja del estudio y el diseño del estudio que no incorporar un grupo control. Por tanto, hay que tomar los resultados con precaución a la espera de confirmarlos en posteriores estudios. 


\section{CONCLUSIONES}

Los resultados de este primer estudio con el programa de realidad virtual Inclúyete VR son prometedores y muestra que puede ser una herramienta que pueden utilizar los profesionales y estudiantes para familiarizarse más con la salud mental y sensibilizarse hacia el estigma en estos trastornos. Se requiere no obstante llevar a cabo estudios controlados que confirmen estos resultados y nos permitan avanzar en la mejora de estas herramientas de intervención.

\section{Agradecimientos}

El presente trabajo ha sido financiado con una ayuda por parte del Ministerio de Ciencia, Innovación y Universidades (Ref.: PSI2017-84961-R AEI/FEDER, UE).

\section{REFERENCIAS BIBLIOGRÁFICAS}

Cangas, A.J., Navarro, N., Aguilar-Parra, J.M., Ojeda, J.J., Cangas, D., Piedra, J.A. y Gallego, J. (2017). StigmaStop: A serious game against the stigma in mental health in educational settings. Frontiers in Psychology, 8 , 1385.

Cangas, A.J., Navarro, N., Aguilar, J.M., Trigueros, R., Gallego, J., Zárate, R. y Gregg,

M. (2019). Usefulness of a serious game to raise awareness among young people about mental health problems. Journal of Clinical Medicine, 8, 1504.

Corrigan, P.W., River, L.P., Lundin, R.K., Penn, D.L., Uphoff-Wasowski,K., Campion,J., Mathisen, J., Gagnon, C., Bergman,M., Goldstein, H. y Kubiak MA. (2001). Three Strategies for Changing Attributions about Severe Mental IIIness. Schizophrenia Bulletin, 27, 187-195.

Corrigan, P.W. y Watson, A.C. (2005). Findings from the National Comorbidity Survey on the frequency of dangerous behavior in individuals with psychiatric disorders. Psychiatry Research, 136, 153-162.

Fresán, A., Robles, R., de Benito, L., Saracco, R. \& Escamilla, R. (2010). Development and psychometric properties of a brief instrument to measure the stigma of aggressiveness in schizophrenia. Actas Españolas de Psiquiatría, 38, 340-4.

Granados-Gámez, G., López-Rodríguez, M., Corral-Granados, A. y Márquez-Hernández,

V.V. (2016). Attitudes and Beliefs of Nursing Students Toward Mental Disorder: The Significance of Direct Experience with Patients. Perspectives in Psychiatric Care, 53, 135-143.

Hansson, L., Jormfeldt, H., Svedberg, P. y Svensson, B. (2013). Mental health professionals attitudes towards people with mental illness: Do they differ from attitudes held by people with mental illness? International Journal of Social Psychiatry, 59, 48- 54.

Horsfall, J., Cleary, M. y Hunt, G.E. (2010). Stigma in mental health: clients and professionals. Issues in Mental Health Nursing, 31, 450-455.

Hortensius R, Neyret S, Slater M, de Gelder B (2018) The relation between bystanders' behavioral reactivity to distress and later helping behavior during a violent conflict in virtual reality. PLOS ONE, 13, e0196074.

Longdon, E. y Read, J. (2017). People with problems, not patients with illnesses: using psychosocial frameworks to reduce the stigma of psychosis. The Israel Journal of Psychiatry and Related Sciences, 54, $24-28$.

Mårtensson, G., Jacobsson, J.W. y Engström, M. (2014). Mental health nursing staffs attitudes towards mental illness: Ananalysis of related factors. Journal of Psychiatric and Mental Health Nursing, 21,782-788.

Mullor, D., Sayans, P., Cangas, A.J. y Navarro, N. (2019). Effect of a serious game (Stigma-Stop) on reducing stigma among psychology students: A controlled study. Cyberpsychology, Behavior, and Social Networking, 22, 205-211.

Saavedra, J., Arias-Sánchez, S., Corrigan, P., \& López, M. (2020). Assessing the factorial structure of the mental illness public stigma in Spain. Disability and Rehabilitation, 1-7.

Wahl, 0. y Aroesty-Cohen, E. (2010). Attitudes of mental health professionals about mental illness: A review of the recent literature. Journal of Community Psychology, 38, 49-62. 
